\title{
Dynamics connect substrate recognition to catalysis in protein kinase A
}

\author{
Larry R. Masterson ${ }^{1,2}$, Cecilia Cheng ${ }^{3}$, Tao $\mathrm{Yu}^{2}$, Marco Tonelli ${ }^{4}$, Alexandr Kornev ${ }^{3}$, Susan \\ S. Taylor ${ }^{3, *}$, and Gianluigi Veglia ${ }^{1,2,{ }^{*}}$ \\ ${ }^{1}$ Department of Biochemistry, Molecular Biology, and Biophysics, University of Minnesota, \\ Minneapolis, Minnesota 55455-0431 \\ ${ }^{2}$ Department of Chemistry, University of Minnesota, Minneapolis, Minnesota 55455-0431 \\ ${ }^{3}$ Department of Chemistry and Biochemistry, University of San Diego, San Diego CA 92093-0654 \\ ${ }^{4}$ National Magnetic Resonance Facility at Madison, Department of Biochemistry, University of \\ Wisconsin-Madison, Madison, Wisconsin 53706-1544
}

\begin{abstract}
Atomic resolution studies of protein kinases have traditionally been carried out in the inhibitory state, limiting our current knowledge on the mechanisms of substrate recognition and catalysis. Using NMR, x-ray crystallography, and thermodynamic measurements we analyzed the substrate recognition process of cAMP-dependent protein kinase (PKA), finding that entropy and protein dynamics play a prominent role. The nucleotide acts as a dynamic and allosteric activator by coupling the two lobes of apo PKA, enhancing the enzyme dynamics synchronously, and priming it for catalysis. The formation of the ternary complex is entropically driven and NMR spin relaxation data reveal that both substrate and PKA are dynamic in the closed state. Our results show that the enzyme toggles between open and closed states, which indicate that a population shift/conformational selection rather than an induced-fit mechanism governs substrate recognition.
\end{abstract}

\section{Keywords \\ Protein Kinase A; Phospholamban; Allostery; Substrate Recognition; Conformational Selection; Intrinsically Disordered Proteins; Membrane Proteins}

\section{Introduction}

Phosphorylation via cAMP-dependent protein kinase A (PKA) is a ubiquitous signaling mechanism that regulates many cellular processes ${ }^{1}$. PKA targets a diverse array of substrates, including proteins localized in the cytoplasm, mitochondria, plasma membrane, sarcoplasmic reticulum membrane, nucleus, microtubules, and actin filaments ${ }^{2}$. The architecture of this enzyme consists of a bean-shaped core which is conserved throughout the protein kinase family ${ }^{3}$. This core has two lobes which flank the active site: a small lobe

\footnotetext{
*To whom correspondence should be addressed: Dr. Gianluigi Veglia, Department of Chemistry and Department of Biochemistry, Molecular Biology, and Biophysics, 231 Church St. SE, Minneapolis, MN 55455. Telephone: (612) 625-0758. Fax: (612)-625-5780. vegli001@umn.edu. Dr. Susan S. Taylor, Department of Chemistry and Biochemistry, University of California- San Diego, 9500 Gilman Dr. CMM W 125, La Jolla, CA 92093. Telephone: (858) 534-3677. Fax: (858) 534-8193. staylor@ucsd.edu.

Author Contributions: L.R.M, C.C, T.Y., M.T., A.K., S.S.T., and G.V. designed experiments, analyzed data and wrote the paper, and L.R.M, C.C., T.Y., and M.T. performed experiments.

There are no competing financial interests.
} 
formed by $\beta$-strands at the $\mathrm{N}$-terminus, which is primarily associated with binding and positioning ATP, and a large lobe that provides a docking surface for substrates or inhibitor proteins ${ }^{4}$. Most of the atomic resolution information on the catalytic subunit of PKA (PKAC) is derived from x-ray crystallographic studies, which mapped three major forms: apo (open state), nucleotide bound (intermediate state or binary complex), and nucleotide and inhibitor bound (closed state or ternary complex) ${ }^{5}$. The crystal structures of the closed state, which mimics the Michaelis complex, has only been captured with kinase inhibitors (peptides or drugs) and in excess (inhibitory) concentrations of $\mathrm{Mg}^{2+}$ ions, precluding our understanding of the substrate recognition process. This problem is common to all protein kinases, whose crystal structures are trapped in inactive states with potent inhibitors. More importantly, there is scarce information on protein kinase dynamics ${ }^{5,6}$ - the equilibrium fluctuations that enable the exploration of the free energy landscape. These fluctuations influence enzymatic reactions since they are responsible for molecular recognition events involved in enzyme-ligand binding ${ }^{7-13}$, or can regulate the catalytic cycle by limiting access to certain conformations which allow turnover ${ }^{12,14-16}$.

Using a combination of NMR spectroscopy and x-ray crystallography, we provide a vivid picture of PKA-C substrate recognition along the reaction coordinates for the open, intermediate, and closed conformational states, mapping out the dynamic landscape on fast (ps-ns) and slow ( $\mu \mathrm{s}-\mathrm{ms})$ time scales. As a substrate, we used a peptide corresponding to the cytoplasmic domain of phospholamban (PLN), a single-pass membrane protein that inhibits the sarcoplasmic reticulum membrane calcium ATPase (SERCA) ${ }^{17}$. We found that nucleotide binding promotes synchronous motions among residues surrounding the active site that correlate with opening and closing of the enzyme's active site cleft. In the ternary complex, some of these dynamics are redistributed, but motions persist around the active site. While this dynamics is not correlated to the chemical step (phosphoryl transfer), it occurs on the same timescale as the rate-determining step of enzyme turnover ${ }^{18}$. Both NMR spectroscopy and $\mathrm{x}$-ray crystallography indicated that the substrate, PLN, adopts an extended and dynamic conformation at the binding groove. The presence of dynamics in the substrate and at the active site of the enzyme can not conform to an induced-fit recognition model, but rather exemplified the conformational selection model of recognition ${ }^{7,19}$. Thermodynamic analysis confirmed that the binding events are entropically driven, providing further support for this mechanism. The coordinated motions that open and close the cleft of the enzyme underscore the role of conformational dynamics in the slow step of catalysis.

\section{Results}

\section{Malleability of the x-ray ternary complex}

Very few protein kinases have been crystallized with natural peptide substrates, and even fewer with protein substrate ${ }^{20}$. Here, we were able to crystallize PKA-C in complex with $5^{\prime}$-adenylyl- $\beta, \gamma$-imidodiphosphate (AMP-PNP) and a substrate peptide at $2.8 \AA$ resolution (Fig. 1 a,b and Supplementary Table 1 online). The peptide we used corresponded to residues 1-19 of PLN (PLN $\left.{ }_{1-19}\right)^{21}$, which comprises the PKA-C recognition site $\left(\mathrm{R}_{13} \mathrm{RAST}_{17}\right)$. We found that this peptide is phosphorylated by PKA-C as efficiently as the full-length protein in isotropic lipid bicelles (Supplementary Fig. 1a,b online), constituting a good mimic of PLN. Although the complex was crystallized under saturating ligand concentrations, two different conformations of the enzyme were present in the asymmetric

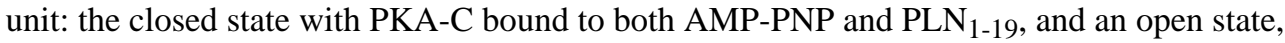
free of bound ligands (apo) (Fig. 1a). In the apo PKA-C molecule, the large lobe is packed against the active site face of the substrate-bound molecule, with $\mathrm{PLN}_{1-19}$ sandwiched between the two PKA-C molecules (Fig. 1a and Supplementary Fig. 2a,b online). The apo 
conformation of PKA-C trapped in this complex is consistent with a previous apo structure ${ }^{3}$, where both lobes of the enzyme are disengaged from each other.

In the ternary complex, the $\mathrm{PLN}_{1-19}$ recognition sequence (residues 12-17) is clearly positioned in the active site groove between the lobes of PKA-C (Fig. 1b). As expected for a catalytically competent conformation, the P-site hydroxyl group of Ser16 in $\mathrm{PLN}_{1-19}$ is aligned to accept the $\gamma-\mathrm{PO}_{4}$ from AMP-PNP, similar to what is observed in the PKA-C crystal structure mimicking the transition $\operatorname{state}^{3}$ (Supplementary Fig. 2a online). The electron densities of $\mathrm{PLN}_{1-19}$ side chains were well defined at the active site, with a clear network of ionic interactions at the recognition $\operatorname{site}^{3}$ (Supplementary Fig. 2b online). Specifically, the P-2 and P-3 arginine residues in $\mathrm{PLN}_{1-19}$ may form hydrogen bonds with Glu127 and Glu230/Glu170 in PKA-C, respectively, in a manner similar to PKA:inhibitor structures $^{5}$. In addition, two key backbone interactions between the $\mathrm{P}-2$ and the $\mathrm{P}+1$ sites of $\mathrm{PLN}_{1-19}$ and Lys168 and Gly200 of PKA-C serve to position the substrate in the binding groove.

The crystal structure of the ternary complex indicated dynamic disorder at the small lobe when PKA-C is bound to the phosphorylatable substrate, $\mathrm{PLN}_{1-19}$. PKA-C was missing electron density for parts of the $\mathrm{N}$ - (residues 1-16) and C-termini (residues 339-342), as well as the conserved glycine-rich loop (residues 47 and 52-54) which positions the $\gamma$-phosphate. The unstructured substrate was also missing the first four residues at the $\mathrm{N}$-terminus and the last two residues at the C-terminus. Strikingly, the B-factors of the small lobe in the ternary complex were significantly higher than in the apo form from the same asymmetric unit (Supplementary Fig. 3a,b online). These data indicated higher malleability in the closed state relative to the open, unligated state of PKA-C.

\section{Substrate recognition is cooperative and entropy driven}

Isothermal titration calorimetry (ITC) measurements showed that $\mathrm{PLN}_{1-19}$ binds to PKA-C with a weak dissociation constant $K_{\mathrm{d}} \sim 50 \mu \mathrm{M}$ (Table 1 and Supplementary Fig. 4a,b online). The binding to $\mathrm{PLN}_{1-19}$ was cooperative with the nucleotide, which caused a five-fold increase in binding affinity. The cooperative effect was slightly larger than the one measured for Kemptide, a minimal recognition sequence for PKA-C ${ }^{22}$. The analysis of the titration curves shows that binding was entropically driven, with an enthalpic contribution of $-1.2 \pm$ $0.1 \mathrm{kcal} \mathrm{mol}^{-1}$ and an entropic penalty of $-5.7 \mathrm{kcal} \mathrm{mol}^{-1}$ (Table 1). This entropically favored binding event is not characteristic of an induced fit mechanism, since enhanced ordering (driven by enthalpy from favorable intermolecular interactions) would be expected ${ }^{7,7}$. As indicated by the high B-factors and absence of electron density from the glycine-rich loop of the x-ray crystal structure, no significant ordering appeared to be induced in the ternary complex.

NMR analysis of amide backbone resonances for isotopically labeled PLN peptide $\left(\mathrm{PLN}_{1-20}\right)$ showed negative $\mathrm{H}-\mathrm{X}$ NOE values and uniformity of the ratio of the transverse and longitudinal relaxation rates $\left(R_{2} / R_{1}\right)$ (Fig. 2a,b), which indicate that when free in solution the peptide is flexible, monomeric, and reorients relatively fast in the NMR time scale $^{23}$. Upon saturation with PKA-C, the majority of H-X NOE values detected across the backbone remain low (near zero), and only residues 13-17 (comprising the recognition sequence) show enhanced rigidity. Although an overall enhancement of rigidity is detected after recognition, the average $\mathrm{H}-\mathrm{X}$ NOE value is 0.54 , characteristic of mobility for these amides. The calculated $R_{2} / R_{1}$ ratios indicate that residues $10-18$ tumble with a correlation time corresponding to a larger macromolecular complex ${ }^{23}$, which only slightly extends this region of interaction. While it is difficult to relate the enhanced structural fluctuations directly with the overall increase in entropy measured by thermodynamic measurements, the NMR and ITC measurements showed a similar trend: NMR measurements suggest an 
increase in configurational entropy upon ligand binding and the ITC reveals that ligand binding is driven by the overall entropy.

\section{Dynamic activation by nucleotide and substrate binding}

The effects of nucleotide and substrate binding on the backbone dynamics of the enzyme were analyzed using nuclear spin relaxation parameters $\left(R_{1}, R_{2}, R_{\mathrm{ex}}\right.$, and $\left.\mathrm{H}-\mathrm{X} \mathrm{NOE}\right)$ for the three major forms of the enzyme ${ }^{5}$ (Supplementary Fig. 5a-c and Supplementary Table 2 online): apo, binary complex, and ternary complex, and these are mapped onto the crystal structure in Figures 3a,b. Our data showed a global decrease in $R_{2} / R_{1}$ ratios upon the transition from apo to the ternary complex (Supplementary Table 2 online), indicating that the entire enzyme is more compact upon ligand binding, in agreement with previous findings ${ }^{3}$. However, changes in the H-X NOE and $R_{e X}$ values in the binary and ternary complexes indicated an increase in backbone dynamics of the enzyme on fast and slow time scales, with a redistribution of motion throughout the backbone.

The apo form is monomeric and well-folded, with a trimmed $\mathrm{H}-\mathrm{X}$ NOE average value of 0.76 (Supplementary Table 2 online) and an effective rotational correlation time of $\sim 19 \mathrm{~ns}$. Using H-X NOE as a proxy for the fast dynamics (ps-ns time scale), we found that the most relevant features are the marked dynamics (NOEs $<0.6$ ) in residues located between structural elements of the enzyme (Fig. 3a and Supplementary Fig. 5a online). In particular, low NOE values were detected for all of the conserved loops, including the glycine-rich, catalytic, DFG, activation, and P +1 loops. Low H-X NOE values were also detected for residues within the acidic cluster (residues 328-334). In this region, Y330 forms electrostatic interactions with an essential arginine of the substrate recognition sequence (R13 in $\mathrm{PLN}_{1-20}$ ), while Glu333, Glu332, and Asp329 interact with the glycine-rich loop in the ligand bound structures 5 .

Surprisingly, very little conformational exchange is present in the apo form on the $\mu \mathrm{s}-\mathrm{ms}$ time scale $\left(R_{e X}\right)$ (Fig. 3b and Supplementary Fig. 5a online). The lack of these slow dynamics is also supported by analysis of the NMR inverse peak heights measured at various temperatures (Supplementary Fig. 6a online) ${ }^{24}$. Relatively small $R_{e_{X}}$ values were measured throughout the entire backbone, with the majority of values distributed around zero $^{25}$. Specifically, the small lobe does not show marked conformational exchange. Only a few residues interspersed throughout the large C-lobe show $R_{\mathrm{ex}}>10 \mathrm{~Hz}$.

Saturation of PKA-C with the nucleotide (AMP-PNP) changed the backbone dynamics throughout the enzyme. While a slight overall restriction of the protein backbone motion occured ( 0.03 increase in the average H-X NOE), increases in both fast and slow dynamics were detected for regions at the active site cleft (Fig. 3a,b and Supplementary Fig. 5b online). This was apparent for the glycine-rich and the DFG loops, which are directly involved in nucleotide binding, as well as the activation loop, the $\mathrm{P}+1$ loop, and the acidic cluster (Supplementary Fig. 5b online). Other loops not directly involved in catalysis showed an increase in fast dynamics, such as the loops linking helices F and G. Other segments peripheral to the catalytic loops became more dynamically restricted.

Dynamics on a $\mu \mathrm{s}-\mathrm{ms}$ timescale were pervasive in the presence of nucleotide, indicating conformational fluctuations in the binary complex form (Fig. 3b and Supplementary Fig. 5b online). Large changes in $R_{e X}$ at the active site cleft were observed in the glycine-rich, DFG, activation, and $\mathrm{P}+1$ loops, as well as the $\mathrm{C}$-helix. Interestingly, the $\mathrm{C}$-helix residues lying on the face oriented toward the nucleotide binding pocket showed higher $R_{e X}$ values than those populating the opposite face (Supplementary Fig. 7 online). The conformational exchange rates detected for these residues likely reflect recruitment of the C-helix to the active site, which is a critical part of activation ${ }^{26}$. Conformational exchange is also detected 
in regions far from the active site, such as the segments that flank the G-helix and the segment that links the conserved APE motif to the F-helix (Fig. 3b). The augmentation of dynamics was also propagated toward the C-terminal tail (residues 322-350), whose putative role is to position the small lobe for catalysis ${ }^{5}$.

Upon saturation with the substrate, the ternary complex remained dynamic, with a redistribution of fast dynamics throughout the PKA-C backbone. For the glycine-rich loop, we observed a monotonic increase of fast dynamics, with the average NOE value dropping from $\sim 0.8$ in the apo, to $\sim 0.7$ in the binary form, to $\sim 0.5$ for the ternary complex (Fig. 3a and Supplementary Fig. 5c online). However, a significant reduction in fast dynamics was detected for residues at the active site located in the P +1 , DFG, and activation loops. Subtle changes occurred throughout the enzyme, indicating that the entire backbone dynamics has reorganized after substrate binding. The reorganization of dynamics in the ternary complex was also apparent by the redistribution of $\mu \mathrm{s}-\mathrm{ms}$ conformational exchange for many regions (Fig. 3b). For instance, the conformational exchange detected for the C-helix in the binary form was quenched in the ternary complex, while the residues forming the base of the Cspine (Ile174, Met128, and Met231) became more dynamic.

Large $R_{e X}$ values were observed for residues throughout the small lobe in the ternary complex. Interestingly, $R_{e x}$ values $>25 \mathrm{~Hz}$ were observed in two regions intimately involved in the catalytic cycle, the glycine-rich and activation loops. The observed slow dynamics in these regions may report on the conformational changes required for opening the active site for product release, the proposed rate-limiting step in the enzyme turnover ${ }^{18,27}$.

\section{Dynamics are synchronous with slow step of catalysis}

In order to determine whether the conformational exchange reports on opening and closing of the enzyme, we plotted $R_{e x}$ vs. the squared difference of chemical shifts $\left(\Delta \omega^{2}\right)$ between the apo and ternary complex (open and closed states) (Fig. $4 a, b)^{28}$. In the fast exchange limit, if a set of nuclear spins is affected by the same conformational exchange process (i.e., open to closed state transitions), a linear correlation is expected between $R_{e X}$ and $\Delta \omega^{2}$. Indeed, we found a linear correlation (correlation coefficient of 0.9) for many residues around the active site cleft upon addition of AMP-PNP (Fig. 4c). These residues were located in the conserved glycine-rich, DFG, activation, and $\mathrm{P}+1$ loops, which line the entrance to the enzyme active site. Analogous to triosephosphate isomerase ${ }^{28}$, this linear correlation suggested that the motions of these residues report on the same exchange process (same $k_{\mathrm{ex}}$ ), which was the opening and closing of the enzyme active site. From the slope of the linear plot and the populations of free and bound states, it is possible to determine the exchange constant for the process, $k_{e X}=203 \pm 18 \mathrm{~s}^{-1}$. This process was much slower than that of ligand (AMP-PNP) exchange between the free and bound states $\left(k_{e x}=k_{o n}\right.$ [AMP$\mathrm{PNP}]+k_{\text {off }}$, which we obtained from NMR lineshape analysis $\left(k_{e x} \sim 3.4 \times 10^{4} \mathrm{~s}^{-1}\right)^{22}$ (Table 1 ) and independently from stopped-flow kinetic measurements ${ }^{18}$. Under these conditions, $k_{\text {off }}$ of AMP-PNP is $\sim 100 \mathrm{~s}^{-1}$, while the opening rate constant determined by the relaxation experiments is ten times slower $\left(\sim 10 \mathrm{~s}^{-1}\right)$. A linear correlation between $R_{e X}$ and $\Delta \omega^{2}$ (correlation coefficient of 0.8 ) was also obtained for many of the same residues for the ternary complex (Fig. 4b). The $k_{e x}$ in this case is $628 \pm 50 \mathrm{~s}^{-1}$, with $k_{\text {open }}=31 \pm 3 \mathrm{~s}^{-1}$. This was similar to the $k_{c a t}$ we measured for phosphorylation of $\mathrm{PLN}_{1-20}$ using a coupled enzyme assay $\left(\sim 23 \mathrm{~s}^{-1}\right)$, indicating that the slow step of catalysis is dominated by the opening and closing of the enzyme ${ }^{18,29}$.

\section{Allosteric network of PKA-C shows non-contiguous pathways}

The dynamics induced by ligand binding proceeded through contiguous paths throughout the active site (Figs. $3 \mathrm{~b}$ and $4 \mathrm{c}$, residues colored in orange), where nearby residues are within 
van der Waals radii contact. However, non-contiguous effects occurred in remote regions of the enzyme (Fig. 4d, residues colored in red, Supplementary Fig. 8 online) which were not synchronous with the opening and closing motions. This dynamics is likely to report on conformational fluctuations other than the toggling between open and closed states and underscores the complexity of the allosteric network within the enzyme and its ability to funnel into different states. Other functional events such as binding to A-kinase anchoring proteins (AKAPs) and/or regulatory subunits may be correlated to these motions. In fact, a number of residues dynamically activated in the ternary complex are distal from the substrate binding site and make close intermolecular contacts upon assembly with the type-I regulatory subunit ${ }^{30}$ (Fig. $4 \mathrm{~d}$ ). Therefore the complexity of the dynamics we observed throughout the backbone of PKA-C could be attributed to its ability to act as a scaffold for many other interactions with it in the cell.

\section{Discussion}

The enzymatic mechanism of phosphoryl transfer for PKA-C has been extensively studied $^{18,29}$. Catalysis by PKA-C comprises three major steps: ligand (ATP and substrate) binding, chemical step (phosphoryl transfer), and product release. The chemical step is fast, while the product release constitutes the rate-determining step of the catalytic cycle ${ }^{18}$. It has been hypothesized that the latter depends on the conformational transitions of the enzyme to eject both the product and $\mathrm{ADP}^{18}$. Previous x-ray studies ${ }^{3,5}$, fluorescence spectroscopy ${ }^{29}$, as well as H/D exchange coupled to mass spectrometry ${ }^{31}$ aimed to understand the enzyme conformational dynamics along the enzymatic cycle. These studies suggest that the apo form becomes more compact as it transitions to the binary and ternary complexes. However, the binary and ternary complexes were studied in the presence of peptide inhibitors and excess concentrations of $\mathrm{Mg}^{2+}$. As a result, it has been proposed that substrate binding by PKA-C proceeds via an induced-fit mechanism with a global rigidification of the enzyme ${ }^{32}$.

In the induced-fit mechanism, significant ordering occurs at the active site resulting in an enthalpically driven binding process ${ }^{7}$. In contrast, our thermodynamics measurements show that the free energy of binding is dominated by a negative overall entropic penalty for the formation of the ternary complex. Moreover, the NMR data revealed substantial backbone dynamics at the active site of the enzyme was induced upon nucleotide binding. This dynamics was not quenched upon substrate binding, indicating a significant contribution of the conformational entropy to the overall entropy for binding. We also observed a preexisting equilibrium of open and closed states in the binary complex prior to formation of the ternary complex (Figs. 4a,b). This supports that the recognition of $\mathrm{PLN}_{1-20}$ is entropically driven and governed by conformational selection rather than an induced fit mechanism ${ }^{7}$.

The dynamic nature of the ternary complex is complemented by the x-ray crystal structure, where electron density is absent or poor for several dynamic regions highlighted by NMR. These regions are located in the enzyme (particularly the glycine-rich loop at the active site) and substrate (terminal regions). This phenomenon may explain why, in general, it has been so difficult to trap peptide substrates in crystal structures with PKA-C. However, it should be noted that substrate recognition by this enzyme appears to be quite complex and is likely influenced by how the enzyme is funneled into a particular functional state. In fact, much stronger binding peptides or drugs which inhibit PKA-C may invoke an induced-fit mechanism upon binding, where increased order and decreased in dynamics place the enzyme in an inhibitory state. This is likely the case of the inhibitor $\mathrm{PKI}_{5-24}$ which crystallized with PKA-C with a well-defined active-site. 
Based on the crystal structures and our current NMR analysis, it is possible to propose a general mechanism for events leading to catalysis. We propose that formation of the Michaelis complex in PKA-C occurs mostly through a population shift/conformational selection mechanism, in which both open and closed states are populated by the enzyme, and ligand binding changes the population of these states. The apo enzyme is dynamically inert in the $\mu \mathrm{s}-\mathrm{ms}$ time scale, with the two lobes of the enzyme disengaged (Fig. 5). This is supported by the absence of conformational exchange detected by NMR and the relatively low B-factors of the apo enzyme crystal structure from this study. Since we observe a linear behavior for Rex and the $15 \mathrm{~N}$ chemical shift changes between the closed and open states, the apo enzyme likely contains very small populations of closed conformations, which were not detected since they may occur on a timescale that the NMR measurements were not able to probe. Nucleotide binding activates the enzyme both dynamically and allosterically. The nucleotide completes the $\mathrm{C}$-spine architecture ${ }^{4}$ and engages the small and large lobes. This interaction functions as a pivot point for the two domains and induces the $\mu \mathrm{s}-\mathrm{ms}$ conformational dynamics detected by NMR (Fig. 5). Subsequent changes in conformation and dynamics upon nucleotide binding prime the active site for catalysis. These changes are not likely to occur without the completion of the $\mathrm{C}$-spine, as shown by a number of mutations, which disrupted this region and led to decreased catalytic activity 4 . Crystallization of one of these mutated enzymes resulted in an open state under conditions where a closed state is typically observed ${ }^{33}$. In our work, we observe opening and closing of the enzyme in the presence of the nucleotide. This opening/closing equilibrium affords the enzyme more effective substrate binding (Fig. 5), which results in the cooperative enhancement in the binding affinity we measured.

An outstanding question which we addressed in this study is whether the backbone dynamics of the ternary complex correlates with any of the catalytic steps. To answer this, we analyzed all of the residues that experience conformational exchange in the ternary complex. We found that the highly conserved loops (glycine-rich, DFG, activation, and P + 1 loops) as well as other catalytically important regions of the complex open and close in a synchronous manner. Moreover, the rate constant for opening the cleft $\left(k_{\text {open }}\right)$ measured in the ternary complex correlates well with the $k_{c a t}$ measured for $\mathrm{PLN}_{1-20}$ phosphorylation. The presence of these dynamics in the ternary complex could constitute an entropic driving force for the enzyme to release the products once phosphorylation occurs, a process which has been identified as the slow-step in catalysis ${ }^{18}$. Therefore, the conformational fluctuations of the ternary complex probed by NMR correlate well with motions that limit enzyme turnover.

While there is still an ongoing debate on the role of dynamics in the chemical step of enzyme catalysis ${ }^{14,34,35}$, recent reports show a prominent role of conformational dynamics in substrate recognition and product release $\mathrm{e}^{9,11-13,19,36}$. Perhaps nowhere is this more apparent than in the highly regulated protein kinases. This conformational dynamics acts locally and allosterically to tune the affinity and selectivity of enzymes, signaling proteins, and receptors ${ }^{8,10,19,37-41}$. In the case of PKA-C, the entire enzyme (catalytic loops, signal integration, and core elements) is dynamic in all the major forms (apo, binary complex, and ternary complex) that mimic the transitions through the catalytic cycle. This dynamics keeps the enzyme from falling into inert (dynamically uncommitted) states. The latter is reminiscent of the energetic counterweight hypothesis proposed for the adenylate kinase ${ }^{42}$.

When bound, the substrate is also quite dynamic, possibly underlying a reciprocal dynamic adaptation between the two binding partners: although their conformations are molded into one another, they remain dynamic prior to the catalytic event. This implies that the Michaelis complex trapped in this study is not a tight structural complex (enthalpy driven), but remains dynamically loose (entropically driven) ${ }^{43}$ as suggested earlier ${ }^{29}$, and undergoes motions synchronous with the slow step of catalysis. This reciprocal dynamic adaptation of 
substrate and enzyme might be a key for the ability of PKA-C to recognize a variety of substrates that typically exist as part of a macromolecular complex.

Recent papers highlight the importance of conformational entropy to the overall entropy of binding for both peptide substrates and drugs ${ }^{9,44}$. For protein kinases, the role of conformational entropy in the context of drug design is relatively unexplored. The results presented here indicate that conformational entropy weighs significantly in the overall free energy of binding and must be taken in consideration for protein kinase drug design.

\section{Methods}

\section{Sample preparation}

PKA-C was expressed and purified as described previously ${ }^{45}$ and PLN $_{1-20}$ was expressed as in reference ${ }^{21}$. Peptide synthesis of PLN $_{1-19}$ was performed using standard Fmoc chemistry on an Applied Biosystems Pioneer or CEM Liberty/Discover microwave synthesizer (for details see Supplementary Information) and HPLC purified using a semi-preparative C18 reversed-phase cartridge (Waters). Purities of pooled fractions were $>95 \%$ as assessed by analytical HPLC (Vydac) and verified by ESI-MS (calculated $2252.6 \mathrm{~m} / \mathrm{z}$, found $2251.8 \mathrm{~m} /$ z). Activity of PKA-C was determined using steady-state enzymatic phosphorylation of Kemptide measured spectrophotometrically at 299 K (see details in the Supplementary Methods). Steady-state phosphorylation of full-length PLN and PLN 1-19 $_{19}$ was performed similarly and described in the Supplementary Methods.

\section{X-ray Crystallography}

The PKA-C:AMP-PNP:PLN ${ }_{1-19}$ complex was obtained by combining a 1:10:10:10 molar ratio mixture of PKA-C (7 mg/ml), $\mathrm{PLN}_{1-19}, \mathrm{MgCl}_{2}$, and AMP-PNP in $20 \mathrm{mM}$ sodium acetate (pH 6.5), $180 \mathrm{mM} \mathrm{KCl}$, and $5 \mathrm{mM}$ DTT. Crystals were harvested, transferred to mother liquor consisting of $15 \%$ glycerol, then flash-frozen in liquid nitrogen. X-ray diffraction data was collected at The Advanced Light Source (Lawrence Berkeley National Laboratory, Berkeley, CA) on beamline 2.2.1. Diffraction data were processed and scaled with HKL2000 46 to $2.8 \AA$ resolution. The initial data clearly fit to a primitive hexagonal lattice and the final data was integrated and scaled in the $\mathrm{P}_{1}$ space group. Initial phases were generated by molecular replacement using the coordinates of the PKA-C:ATP:PKI $5-24$ complex without the PKI peptide (PDB code 1ATP) as a search model. The ternary complex crystallized with two molecules in the asymmetric unit (Z-score 26.5 and 38.7) corresponding to a solvent content of 52\%. The first molecule corresponds to a ternary complex containing PKA-C, AMP-PNP/ $\mathrm{Mg}^{2+}$, and $\mathrm{PLN}_{1-19}$ and the second molecule corresponds to the apo form. The final $\mathrm{R}$ and $\mathrm{R}$-free values after data refinement (details in the Supporting Information) were $21.6 \%$ and $28.7 \%$, respectively. The final model contained PKA-C residues 16-350 bound to AMP-PNP, two $\mathrm{Mg}^{2+}$ ions, and PLN residues 5-15 in the first molecule and the apo form of PKA-C with residues 13-350 in the second molecule. Optimization of crystallization conditions and full details of data refinement are provided in the Supplementary Methods.

ITC measurements-ITC data were acquired on a MCS-ITC microcalorimeter (MicroCal Inc., Northampton, MA). Stock solutions of PKA-C and PLN $_{1-19}$ were dissolved in $20 \mathrm{mM}$ phosphate buffer solution ( $\mathrm{pH}$ 6.5) containing $180 \mathrm{mM} \mathrm{KCl}$ and $4 \mathrm{mM} \mathrm{MgCl}_{2}$, and degassed. Titrations were conducted at $27^{\circ} \mathrm{C}$ using $0.1 \mathrm{mM}$ PKA-C in the absence or presence of $6 \mathrm{mM}$ AMP-PNP and with a stock of synthetic $\mathrm{PLN}_{1-19}(1.8 \mathrm{mM})$. The samples were stirred at $410 \mathrm{rpm}$. 20 injections separated by an equilibration period of $300 \mathrm{~s}$ were used ( $5 \mu \mathrm{L}$ for the first, followed by $10 \mu \mathrm{L}$ for each of the remaining). A one-site binding 
model was assumed and the data were fit using MicroCal Origin software (version 5.0) and all data were repeated in triplicate.

NMR spectroscopy and data analysis-Typical NMR samples consisted of $\sim 500 \mu \mathrm{M}$ PKA-C, $10 \mathrm{mM} \mathrm{MgCl}_{2}, 90 \mathrm{mM} \mathrm{KCl}, 20 \mathrm{mM} \mathrm{KH}_{2} \mathrm{PO}_{4}, 10 \mathrm{mM}$ MEGA-8, $20 \mathrm{mM}$ DTT, and $5 \%{ }^{2} \mathrm{H}_{2} \mathrm{O}$ (uncorrected $\mathrm{pH}=6.5$ ). For the binary complex, PKA-C was saturated with $12 \mathrm{mM}$ AMP-PNP, while the ternary complex was obtained by saturating the binary complex with $700 \mu \mathrm{M}$ PLN $_{1-19}$. Solution NMR experiments were carried out on Varian instruments operating at 600.14 or $800.29 \mathrm{MHz}{ }^{1} \mathrm{H}$ Larmor frequency, using an inverse triple axis gradient cryoprobe at $33{ }^{\circ} \mathrm{C}$. The data were processed with NMRPIPE ${ }^{47}$ and analyzed using the software Sparky. Backbone assignments were according to the procedures described in the Supporting Methods. Nuclear spin relaxation experiments were performed according to reference ${ }^{48}$, with TROSY-detection ${ }^{49} . R_{1}, R_{l \rho}$, and H-X NOE experiments for PKA-C $\left(\mathrm{PLN}_{1-20}\right)$ samples were conducted using a spectral width of $10,500(6,500) \mathrm{Hz}$ in the direct detected dimension and 2,200 $(1,200) \mathrm{Hz}$ in the indirect dimension. For $R_{l \rho}$ measurements, a spin-lock field strength of $1,500 \mathrm{~Hz}$ centered at the ${ }^{15} \mathrm{~N}$ carrier frequency was used. $R_{l \rho}$ values were converted into $R_{2}$ as described ${ }^{50} . R_{1}$ and $R_{1 \rho}$, values were calculated b fitting intensities to a single exponential decay and errors were taken from repeats of single data points. $\mathrm{H}-\mathrm{X}$ NOE values were taken as the ratio of intensities from experiments performed with and without saturation. Errors in the $\mathrm{H}-\mathrm{X}$ NOE were calculated via error propagation using root mean square noise of the spectra.

$R_{e X}$ was measured using the method introduced by Palmer ${ }^{25}$, with a Hahn echo period of $2 /$ $J_{\mathrm{NH}}(10.8 \mathrm{~ms})$. Peak intensities were used to determine $R_{e X}$ according to ${ }^{24,25}$ :

$$
R_{e x} \approx C_{z z} \ln \left(\rho_{z z}\right)+C_{\beta} \ln \left(\rho_{\beta}\right)
$$

where $C_{\mathrm{zz}}=(2 \tau)^{-1}, \mathrm{C}_{\beta}=(\langle\kappa\rangle-1)(4 \tau)^{-1}, \kappa=1-2 * \ln \rho_{\mathrm{zz}} / \ln \rho_{\beta}, \rho_{\mathrm{zz}}=I_{z z} / I_{\alpha}$, and $\rho_{\beta}=I_{\beta} / I_{\alpha}$ The intensities of the $a, \beta$, and $z Z$ spin states $\left(I_{a}, I_{b}\right.$, and $\left.I_{z Z}\right)$ were obtained from 2-D experiments recorded in triplicate. The value $\langle\kappa\rangle$ was obtained from the trimmed mean of amides not exhibiting chemical exchange. Errors in $\mathrm{R}_{\mathrm{ex}}$ were calculated by error propagation using root mean square noise of the spectra. The verification of residue-specific $R_{\mathrm{ex}}$ was done by measuring inverse peak heights (Î) at decreasing temperatures ${ }^{24}$. The trimmed mean of resonance peak intensities $(<\triangleright)$ at temperatures of 22,27 and $33{ }^{\circ} \mathrm{C}$ were used to scale individual peak heights $(I)$ to yield $\hat{I}=\langle I / I$. Resonances which experienced conformational exchange diminished in peak intensity more significantly with decreasing temperatures ${ }^{24}$ as shown in the Supporting Information.

The linear correlation of $R_{e X}$ versus $\Delta \omega^{2}$ was determined assuming a two-site fast exchange model (i.e., $\left.k_{e X}>\Delta \omega\right)^{28}$ :

$$
R_{e x}=\frac{p_{f} p_{b} \Delta \omega^{2}}{k_{e x}}
$$

where $p_{f}$ and $p_{b}$ are the fractional populations of free and bound forms at equilibrium, $\Delta \omega$ is the chemical shift difference between the two sites, and $k_{e X}$ is the exchange rate constant (the sum of forward and reverse kinetic rate constants). Analysis of the nucleotide bound dynamics was done using $\Delta \omega$ values for the transition from apo to intermediate forms, and analysis of the ternary state was done using $\Delta \omega$ values for the transition from the apo to ternary complex. The dissociation constants for AMP-PNP and PLN $_{1-20}$ were used to 
determine fractional populations to yield $k_{e x}, k_{\text {open }}$, and $k_{\text {closed }}{ }^{28}$. Errors in these values were propagated from the errors obtained in linear least squares fitting to Equation 2.

\section{Accession codes}

Coordinates and structure factors have been deposited to the Protein Data Bank under the accession number 307L.

\section{Supplementary Material}

Refer to Web version on PubMed Central for supplementary material.

\section{Acknowledgments}

This work was supported by the NIH (GM64742 and HL080081 to G.V, and GM19301 to S.S.T.). NMR data were collected at NMRFAM (NIH: P41RR02301, P41GM66326, RR02781, and RR08438; NSF: DMB-8415048, OIA-9977486, BIR-9214394) and the U. of Minnesota NMR Facility (NSF BIR-961477). We thank J.P. Loria (Yale University) for providing the TROSY Hahn-Echo pulse sequence, and also we would like to thank J. P. Loria and G. Melacini (McGill University) for critical analysis of the paper.

\section{References}

1. Walsh DA, Van Patten SM. Multiple pathway signal transduction by the cAMP-dependent protein kinase. FASEB J. 1994; 8:1227-1236. [PubMed: 8001734]

2. Shabb JB. Physiological substrates of cAMP-dependent protein kinase. Chem Rev. 2001; 101:23812411. [PubMed: 11749379]

3. Taylor SS, Yang J, Wu J, Haste NM, Radzio-Andzelm E, Anand G. PKA: A portrait of protein kinase dynamics. Biochim Biophys Acta. 2004; 1697:259-269. [PubMed: 15023366]

4. Kornev AP, Taylor SS. Defining the conserved internal architecture of a protein kinase. Biochim Biophys Acta. 2009; 1804:440-444. [PubMed: 19879387]

5. Johnson DA, Akamine P, Radzio-Andzelm E, Madhusudan M, Taylor SS. Dynamics of cAMPdependent protein kinase. Chem Rev. 2001; 101:2243-2270. [PubMed: 11749372]

6. Vajpai N, et al. Solution conformations and dynamics of ABL kinase-inhibitor complexes determined by NMR substantiate the different binding modes of imatinib/nilotinib and dasatinib. J Biol Chem. 2008; 283:18292-18302. [PubMed: 18434310]

7. Jarymowycz VA, Stone MJ. Fast time scale dynamics of protein backbones: NMR relaxation methods, applications, and functional consequences. Chem Rev. 2006; 106:1624-1671. [PubMed: 16683748]

8. Popovych N, Sun S, Ebright RH, Kalodimos CG. Dynamically driven protein allostery. Nat Struct Mol Biol. 2006; 13:831-838. [PubMed: 16906160]

9. Marlow MS, Dogan J, Frederick KK, Valentine KG, Wand AJ. The role of conformational entropy in molecular recognition by calmodulin. Nat Chem Biol. 2010; 6:352-358. [PubMed: 20383153]

10. Gsponer J, et al. A coupled equilibrium shift mechanism in calmodulin-mediated signal transduction. Structure. 2008; 16:736-746. [PubMed: 18462678]

11. Yao X, Rosen MK, Gardner KH. Estimation of the available free energy in a LOV2-J alpha photoswitch. Nat Chem Biol. 2008; 4:491-497. [PubMed: 18604202]

12. Mittag T, Kay LE, Forman-Kay JD. Protein dynamics and conformational disorder in molecular recognition. J Mol Recognit. 2009

13. Tzeng SR, Kalodimos CG. Dynamic activation of an allosteric regulatory protein. Nature. 2009; 462:368-372. [PubMed: 19924217]

14. Garcia-Viloca M, Gao J, Karplus M, Truhlar DG. How enzymes work: Analysis by modern rate theory and computer simulations. Science. 2004; 303:186-195. [PubMed: 14716003]

15. Beach H, Cole R, Gill ML, Loria JP. Conservation of mus-ms enzyme motions in the apo- and substrate-mimicked state. J Am Chem Soc. 2005; 127:9167-76. [PubMed: 15969595] 
16. Henzler-Wildman KA, Lei M, Thai V, Kerns SJ, Karplus M, Kern D. A hierarchy of timescales in protein dynamics is linked to enzyme catalysis. Nature. 2007; 450:913-916. [PubMed: 18026087]

17. Traaseth NJ, et al. Structural and dynamic basis of phospholamban and sarcolipin inhibition of ca(2+)-ATPase. Biochemistry. 2008; 47:3-13. [PubMed: 18081313]

18. Adams JA. Kinetic and catalytic mechanisms of protein kinases. Chem Rev. 2001; 101:2271-2290. [PubMed: 11749373]

19. Boehr DD, Nussinov R, Wright PE. The role of dynamic conformational ensembles in biomolecular recognition. Nat Chem Biol. 2009; 5:789-796. [PubMed: 19841628]

20. Mao DY, Ceccarelli DF, Sicheri F. "Unraveling the tail" of how SRPK1 phosphorylates ASF/SF2. Mol Cell. 2008; 29:535-537. [PubMed: 18342599]

21. Masterson LR, et al. Expression and purification of isotopically labeled peptide inhibitors and substrates of cAMP-dependant protein kinase A for NMR analysis. Protein Expr Purif. 2009; 64:231-236. [PubMed: 19027069]

22. Masterson LR, Mascioni A, Traaseth NJ, Taylor SS, Veglia G. Allosteric cooperativity in protein kinase A. Proc Natl Acad Sci U S A. 2008

23. Kay LE, Torchia DA, Bax A. Backbone dynamics of proteins as studied by $15 \mathrm{~N}$ inverse detected heteronuclear NMR spectroscopy: Application to staphylococcal nuclease. Biochemistry. 1989; 28:8972-8979. [PubMed: 2690953]

24. Fenwick MK, Oswald RE. NMR spectroscopy of the ligand-binding core of ionotropic glutamate receptor 2 bound to 5-substituted willardiine partial agonists. J Mol Biol. 2008; 378:673-685. [PubMed: 18387631]

25. Wang C, Rance M, Palmer AG 3rd. Mapping chemical exchange in proteins with MW > $50 \mathrm{kD}$. J Am Chem Soc. 2003; 125:8968-8969. [PubMed: 15369325]

26. Shan Y, et al. A conserved protonation-dependent switch controls drug binding in the abl kinase. Proc Natl Acad Sci U S A. 2009; 106:139-144. [PubMed: 19109437]

27. Lew J, Taylor SS, Adams JA. Identification of a partially rate-determining step in the catalytic mechanism of cAMP-dependent protein kinase: A transient kinetic study using stopped-flow fluorescence spectroscopy. Biochemistry. 1997; 36:6717-6724. [PubMed: 9184152]

28. Massi F, Wang C, Palmer AG 3rd. Solution NMR and computer simulation studies of active site loop motion in triosephosphate isomerase. Biochemistry. 2006; 45:10787-10794. [PubMed: 16953564]

29. Li F, Juliano C, Gorfain E, Taylor SS, Johnson DA. Evidence for an internal entropy contributin to phosphoryl transfer: A study of domain clossure, backbone flexibility, and the catalytic cycle of cAMP-dependent protein kinase. J Mol Biol. 2002; 315:459-469. [PubMed: 11786025]

30. Kim C, Cheng CY, Saldanha SA, Taylor SS. PKA-I holoenzyme structure reveals a mechanism for cAMP-dependent activation. Cell. 2007; 130:1032-1043. [PubMed: 17889648]

31. Yang J, Garrod SM, Deal MS, Anand GS, Woods J, Virgil L, Taylor S. Allosteric network of cAMP-dependent protein kinase revealed by mutation of Tyr204 in the P+1 loop. J Mol Biol. 2005; 346:191-201. [PubMed: 15663937]

32. Hyeon C, Jennings PA, Adams JA, Onuchic JN. Ligand-induced global transitions in the catalytic domain of protein kinase A. Proc Natl Acad Sci U S A. 2009; 106:3023-3028. [PubMed: 19204278]

33. Wu J, et al. Crystal structure of the E230Q mutant of cAMP-dependent protein kinase reveals an unexpected apoenzyme conformation and an extended N-terminal A helix. Protein Sci. 2005; 14:2871-2879. [PubMed: 16253959]

34. Kamerlin SC, Warshel A. At the dawn of the 21st century: Is dynamics the missing link for understanding enzyme catalysis? Proteins. 2010; 78:1339-1375. [PubMed: 20099310]

35. Schwartz SD, Schramm VL. Enzymatic transition states and dynamic motion in barrier crossing. Nat Chem Biol. 2009; 5:551-558. [PubMed: 19620996]

36. Smock RG, Gierasch LM. Sending signals dynamically. Science. 2009; 324:198-203. [PubMed: 19359576]

37. Kumar S, Ma B, Tsai CJ, Sinha N, Nussinov R. Folding and binding cascades: Dynamic landscapes and population shifts. Protein Sci. 2000; 9:10-19. [PubMed: 10739242] 
38. Hammes GG. Multiple conformational changes in enzyme catalysis. Biochemistry. 2002; 41:82218228. [PubMed: 12081470]

39. Hammes-Schiffer S, Benkovic SJ. Relating protein motion to catalysis. Annu Rev Biochem. 2006; 75:519-541. [PubMed: 16756501]

40. Swain JF, Gierasch LM. The changing landscape of protein allostery. Curr Opin Struct Biol. 2006; 16:102-108. [PubMed: 16423525]

41. Das R, Chowdhury S, Mazhab-Jafari MT, Sildas S, Selvaratnam R, Melacini G. Dynamically driven ligand selectivity in cyclic nucleotide binding domains. J Biol Chem. 2009; 284:2368223696. [PubMed: 19403523]

42. Muller CW, Schlauderer GJ, Reinstein J, Schulz GE. Adenylate kinase motions during catalysis: An energetic counterweight balancing substrate binding. Structure. 1996; 4:147-156. [PubMed: 8805521]

43. Grunberg R, Nilges M, Leckner J. Flexibility and conformational entropy in protein-protein binding. Structure. 2006; 14:683-693. [PubMed: 16615910]

44. Mauldin RV, Carroll MJ, Lee AL. Dynamic dysfunction in dihydrofolate reductase results from antifolate drug binding: Modulation of dynamics within a structural state. Structure. 2009; 17:386394. [PubMed: 19278653]

45. Masterson LR, Shi L, Tonelli M, Mascioni A, Mueller MM, Veglia G. Backbone NMR resonance assignment of the catalytic subunit of cAMP-dependent protein kinase A in complex with AMPPNP. Biomol NMR Assign. 2009; 3:115-117. [PubMed: 19636960]

46. Minor W, Tomchick D, Otwinowski Z. Strategies for macromolecular synchrotron crystallography. Structure. 2000; 8:R105-10. [PubMed: 10801499]

47. Delaglio F, Grzesiek S, Vuister GW, Zhu G, Pfeifer J, Bax A. NMRPipe: A multidimensional spectral processing system based on UNIX pipes. J Biomol NMR. 1995; 6:277-293. [PubMed: 8520220]

48. Farrow NA, et al. Backbone dynamics of a free and phosphopeptide-complexed src homology 2 domain studied by 15N NMR relaxation. Biochemistry. 1994; 33:5984-6003. [PubMed: 7514039]

49. Pervushin K, Riek R, Wider G, Wuthrich K. Attenuated T2 relaxation by mutual cancellation of dipole-dipole coupling and chemical shift anisotropy indicates an avenue to NMR structures of very large biological macromolecules in solution. Proc Natl Acad Sci U S A. 1997; 94:12366-71. [PubMed: 9356455]

50. Tjandra N, Wingfield P, Stahl S, Bax A. Anisotropic rotational diffusion of perdeuterated HIV protease from 15N NMR relaxation measurements at two magnetic fields. J Biomol NMR. 1996; 8:273-284. [PubMed: 8953218] 


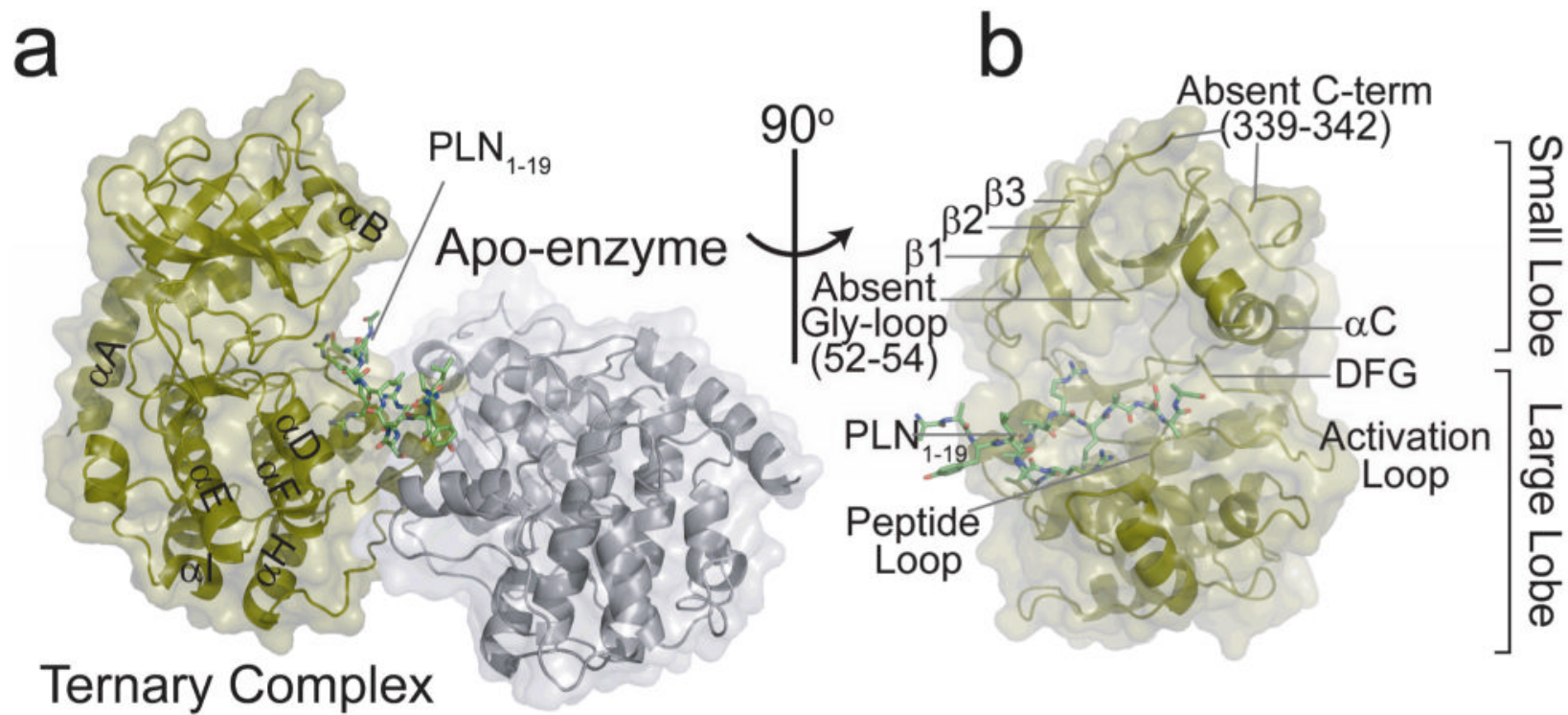

Fig. 1.

X-ray crystal structure of the PKA-C ternary complex containing AMP-PNP and PLN 1 -19. (a) The asymmetric unit revealed two molecules of PKA-C, an apo (open, grey) form and a ternary (closed, tan) complex. (b) The ternary complex is missing the first 15 residues of the $\mathrm{N}$-terminus, part of the glycine-rich loop and C-terminus, which is likely due to conformational disorder. 
a
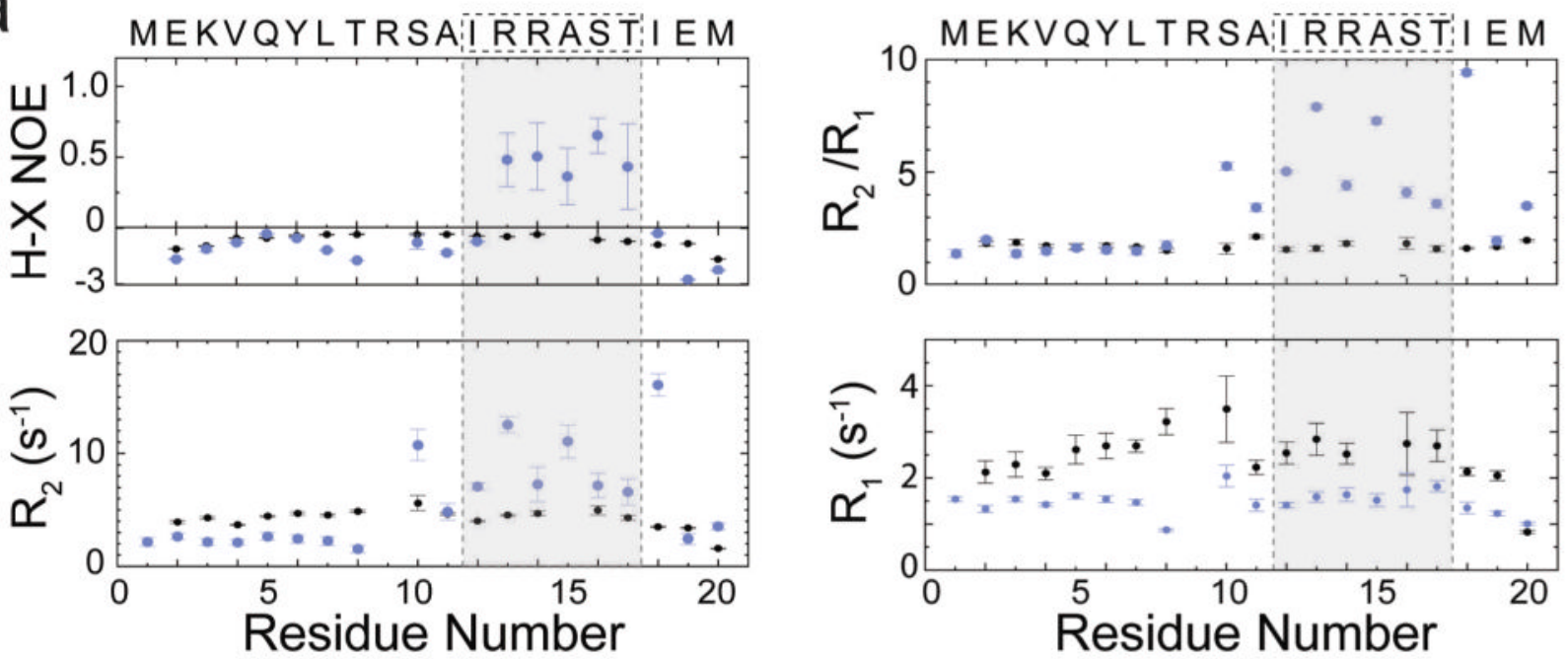

b

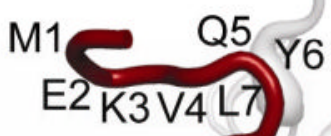

\section{6}

T8

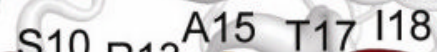
A15 T17 118 E19
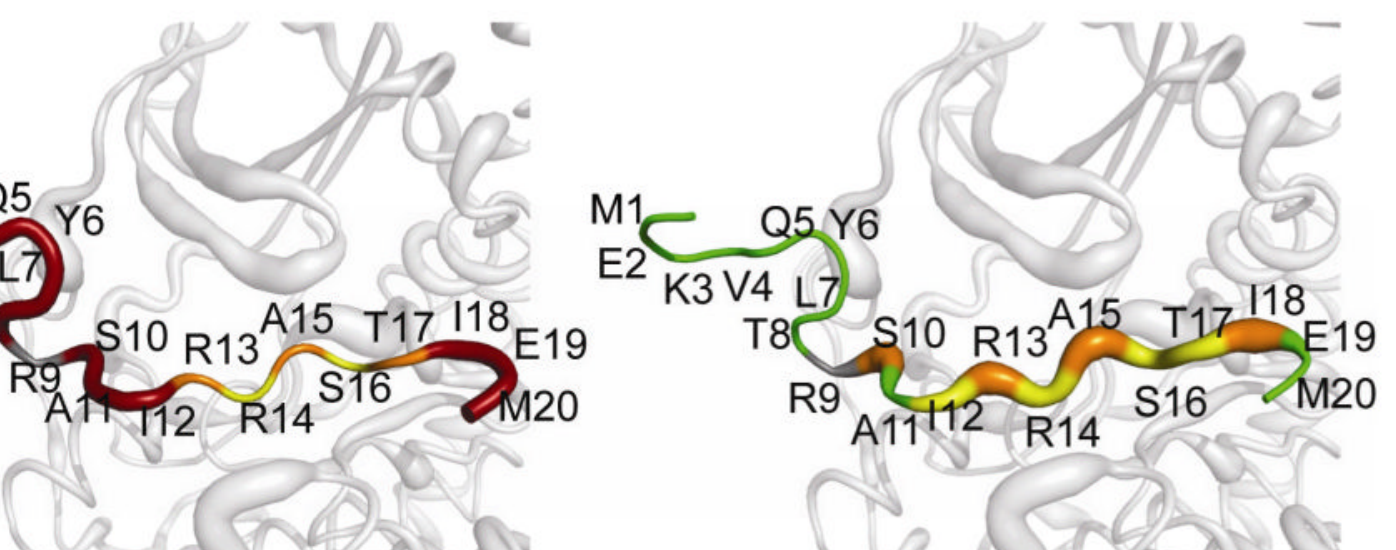

$\left.\begin{aligned} & \text { ய } \\ & \text { Z } \\ & \text { X } \\ & \text { 工 }\end{aligned}\right|_{<0.25} ^{>0.75}$

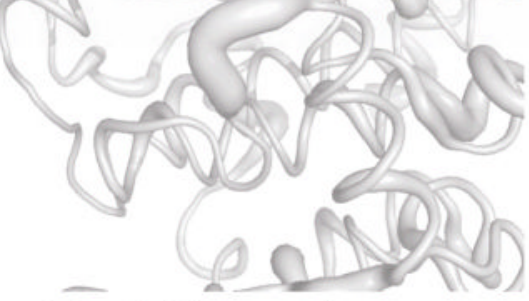

Fast Dynamics

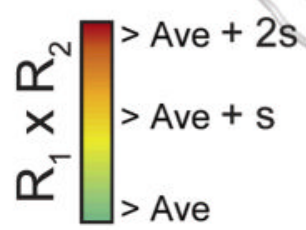

Slow Dynamics

Fig. 2.

Details of the interaction of isotopically labeled PLN $1-20$ with PKA-C. (a) NMR nuclear spin dynamics of $\mathrm{PLN}_{1-20}$ in the free (black) and bound to PKA-C in the presence of AMPPNP (blue). (b) Mapping of these dynamics in the bound form shows that ordering around the recognition sequence was observed, while elevated $R_{2} / R_{1}$ values extend the region of interaction to residues 10-17. Data represent fitted values and associated error from nonlinear fitting. 


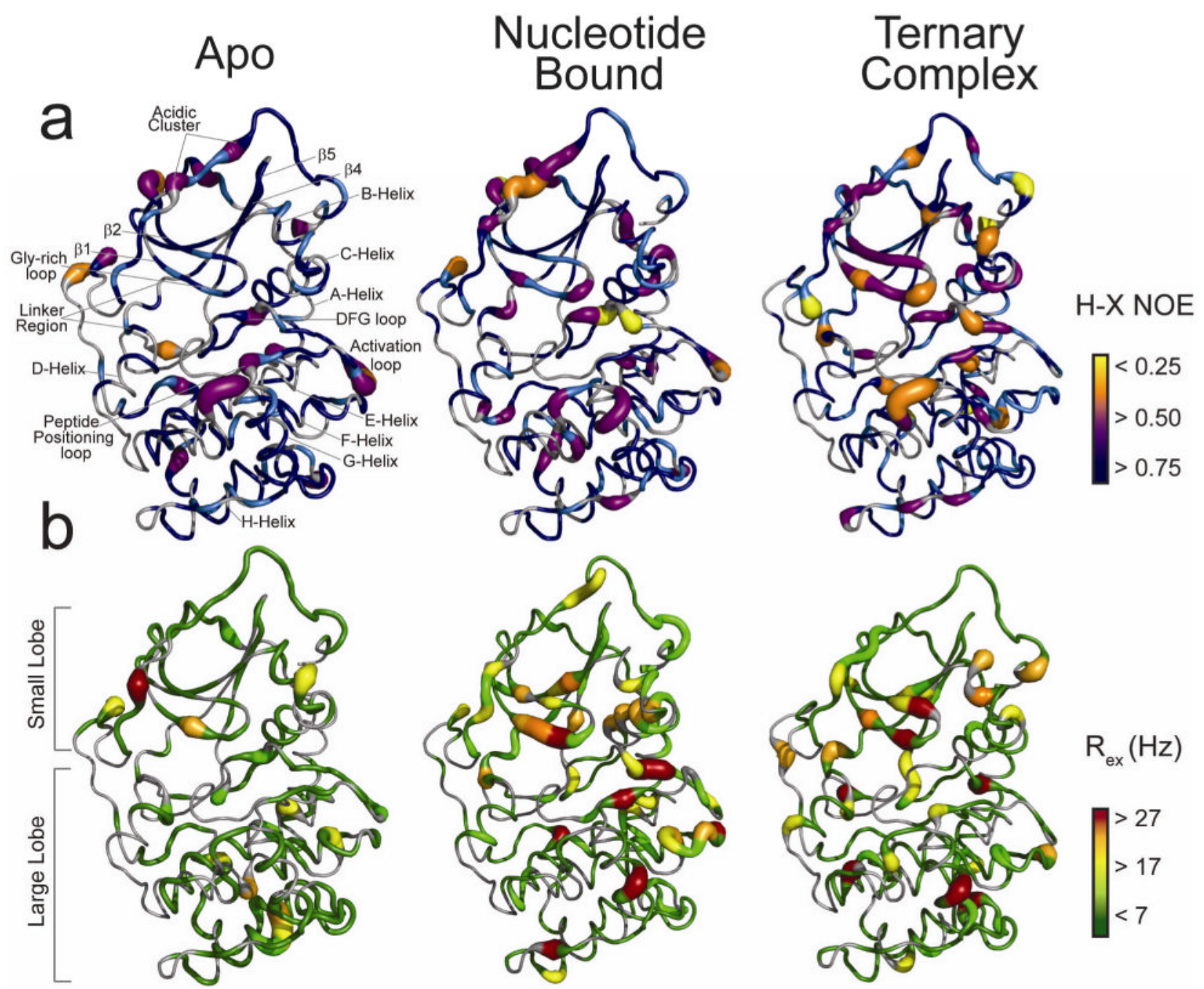

Fig. 3.

Mapping of the backbone amide dynamics of PKA-C from the apo to ternary complex. Structural elements and loops are indicated. Fast (a) and slow (b) dynamics are shown for PKA-C in the apo (left), binary form (middle), and the ternary complex containing AMPPNP and PLN 1-20 $_{\text {(right). }}$ 

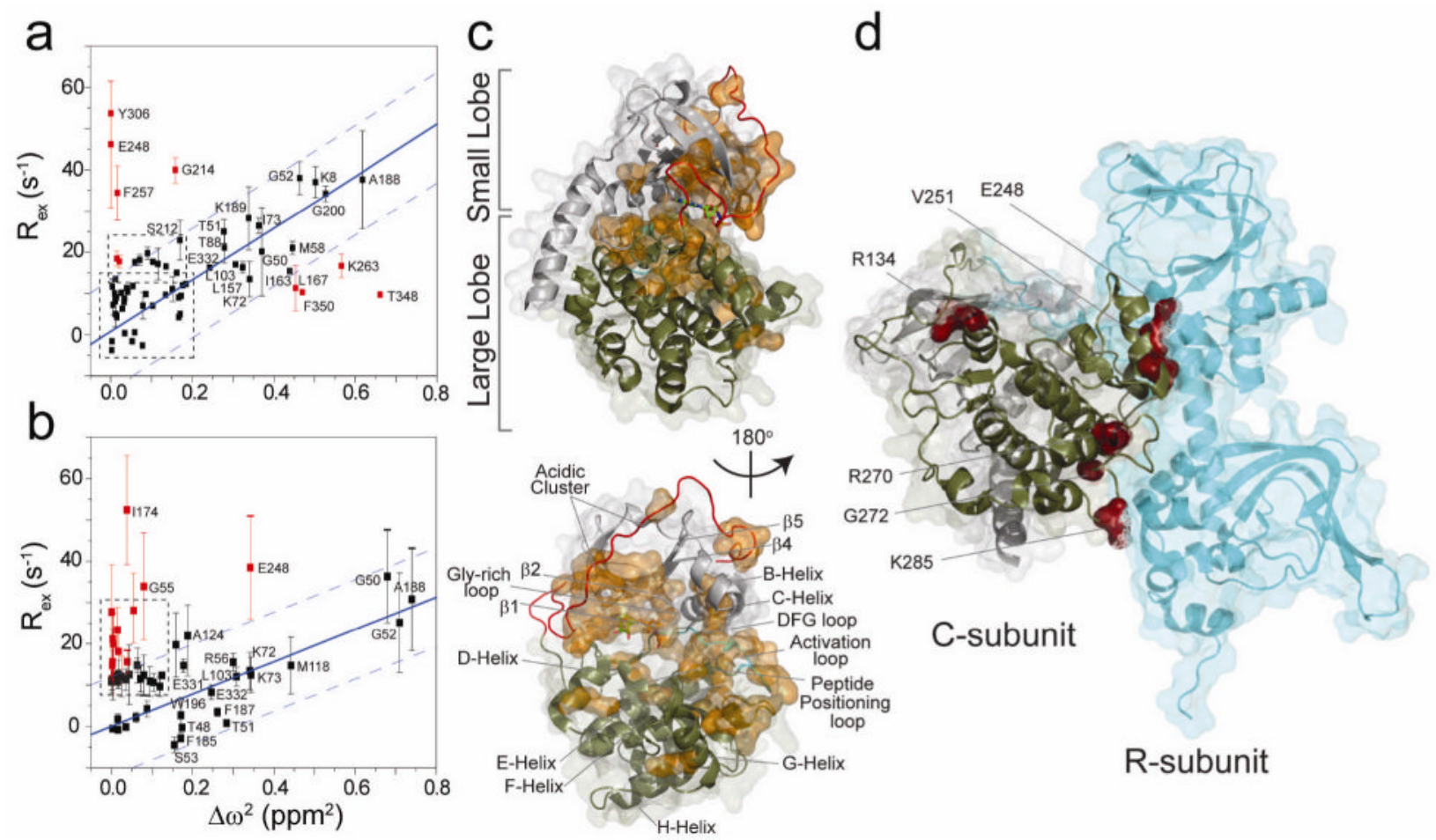

R-subunit

Fig. 4.

Opening and closing of the enzyme active site cleft. Correlation plots of $R_{e X}$ for (a) AMPPNP bound form or (b) PLN $_{1-20}$ /AMP-PNP bound form with the chemical shift differences of open and closed states. (c) The nucleotide induces opening and closing at the entrance of the enzyme which is reported by contiguous and non-contiguos pathways from the active site (residues denoted in orange). Errors in $R_{e_{x}}$ were taken from error propagation of equation 1 using the r.m.s noise of the NMR spectra. (d) In the ternary complex, residues which are not linear with opening and closing are distal and are known to interact with the regulatory subunit based on crystal structure $2 \mathrm{QCS}$. 


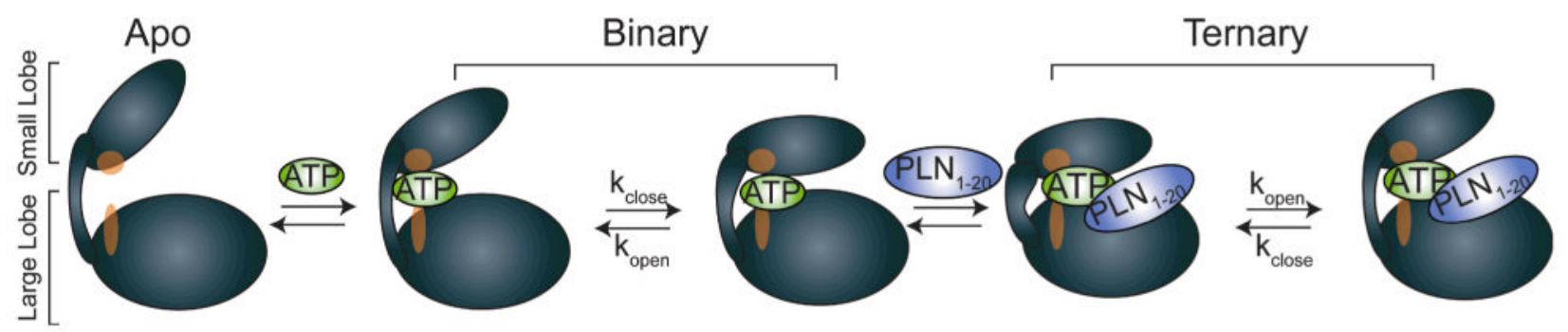

Fig. 5.

Model for the mechanism of the formation of a catalytically competent ternary complex. The apo form contains the $\mathrm{C}$-spine residues (red) which are disengaged from the two lobes. Nucleotide binding completes the $\mathrm{C}$-spine architecture and induces the conformational changes throughout the enzyme. The conformational fluctuations (opening and closing) present in the ternary complex limit the rate of catalysis. 


\section{Table 1}

Thermodynamic parameters for the binding of PKA-C to PLN $\mathrm{PL}_{1-20}$ in the presence or absence of nucleotide. Data represent mean values \pm one s.d. for triplicate measurements.

\begin{tabular}{ccccc}
\hline & $\Delta \mathbf{H}(\mathbf{k c a l} / \mathbf{m o l})$ & $\Delta \mathbf{S}(\mathbf{k c a l} / \mathbf{m o l} * \mathbf{K})$ & $-\mathbf{T} \Delta \mathbf{S}(\mathbf{k c a l} / \mathbf{m o l})$ & $\mathbf{K}_{\mathbf{d}}(\boldsymbol{\mu M})$ \\
\cline { 2 - 5 } Apo form & $-0.6 \pm 0.1$ & $0.017 \pm 0.001$ & $-5.1 \pm 0.2$ & $49 \pm 8$ \\
Binary complex & $-1.2 \pm 0.1$ & $0.019 \pm 0.001$ & $-5.7 \pm 0.1$ & $10 \pm 4$ \\
\hline
\end{tabular}




\section{Table 2}

Observed rate constants measured using the $R_{e X} / \Delta \omega^{2}$ correlation plot or from exchange of nucleotide based on NMR line shape analysis during ligand titration measurements. Values represent fitted values and associated errors from linear least squares analysis (conformational rates, denoted conf) or mean \pm one s.d. for 6 residues (ligand exchange rates, denoted ligand)

\begin{tabular}{lcc}
\hline Observed kinetic constant & Ligand Exchange $^{*}$ & Conformational Exchange \\
\hline $\mathrm{k}_{\mathrm{ex}}\left(\mathrm{s}^{-1}\right)$ & $3.37 \times 10^{6} \pm 0.51 \times 10^{6}$ & $203 \pm 18$ \\
$\mathrm{k}_{\mathrm{off}}^{\text {ligand }}\left(\mathrm{s}^{-1}\right)$ & $109 \pm 4$ & - \\
$\mathrm{k}_{\mathrm{on}}^{\text {ligand }}\left(\mathrm{s}^{-1}\right)^{*}$ & $3.36 \times 10^{6} \pm 0.50 \times 10^{6}$ & - \\
$\mathrm{k}_{\mathrm{open}}^{\mathrm{conf}}\left(\mathrm{s}^{-1}\right)$ & - \\
$\mathrm{k}_{\text {closed }}^{\text {conf }}\left(\mathrm{s}^{-1}\right)$ & $10 \pm 2$ \\
$*$ & \\
The observation of a pseudo-first order rate constant for $k_{\text {On }}$ is expected (i.e., $k_{\text {on }}^{\text {ob }}=k_{\text {on }}[$ Ligand] $)$.
\end{tabular}

